\title{
Ultramicronized Palmitoylethanolamide Reduces Frequency and Pain Intensity in Migraine: A Pilot Study
}

\author{
Giorgio Dalla Volta*, Paola Zavarize, Gaelle FK Ngonga, Daniela Carli
}

Department of Neurology, Clinical Institute of Brescia City, Brescia, Italy

*Corresponding author: Dr Giorgio Dalla Volta, Dipartimento di Neurologia dell'Istituto Clinico Città di Brescia, Via Gualla 15, 25100 BRESCIA, Italy, Tel: +39 030 3757777/ Fax: +39 030 3710444/ Mobile:+39 3355494124; E-mail: dalla@numerica.it

\begin{abstract}
The aim of this open study is to verify whether treatment with ultra-micronized palmitoylethanolamide can reduce headache frequency and intensity in patients suffering from migraine when used in migraine preven-tion. Fifty patients, suffering from migraine without aura, for at least 6 months with a frequency of monthly crises from 3 to 8 and with presence of headache from 4 to 12 days per month, were treated sublingually with ultra-micronized palmitoylethanolamide microgranules $600 \mathrm{mg}$ bid for 3 months. The primary endpoint was reduction in headache days per month; secondary endpoint was duration and intensity of pain crisis and number of analgesics taken per month and changes of thermographic patterns.

All the considered parameters significantly improved at the end of treatment. The mean number of days per month with migraine significantly decreased $(p<0.0001)$; pain intensity was significantly $(p<0.0001)$ miti-gated; the number of analgesics taken significantly decreased and the thermographic patterns showed a reduction of hypothermia as well as the response to trigger factors. No serious adverse events have been observed.

Despite the limitations of the study due to its open-label nature it would be desirable to confirm with further clinical trials the ultra-micronized Palmitoylethanolamide effect in migraine prevention.
\end{abstract}

\section{Introduction}

Migraine is considered one of the most prevalent neurological disorders, affecting about $15 \%$ of the adult population worldwide. The biological origin of migraine remains multifactorial: clinical studies suggest abnormal cortical, cerebrovascular, and immune functions. However, it is now well-accepted that the pain, persistence and throbbing features of migraine are mediated by increased sensitivity (i.e. sensitization) and the ensuing activation of sensory neurons innervating intracranial meninges and their related large blood vessels ${ }^{[1]}$. These are the first-order neurons in the migraine pain pathway.

Given the increasing importance placed on central and peripheral sensitization in the pathogenesis of migraine, the mechanisms of activation of trigeminal sensory afferents take on a central role. Sensitization results from the local release of neuropeptides that cause vasodilatation (mediated by calcitonin gene-related peptide) and an increase in vascular permeability (mediated by substance P and neurokinin A) accompanied by mast cell activation. Mast cells, members of the innate
Received date: August 04, 2015

Accepted date: February 25, 2016

Published date: February 29, 2016

Citation: Dalla Volta, G., et al. Ultramicronized Palmitoylethanolamide Reduces Frequency and Pain Intensity in Migraine: A Pilot Study. (2016) J Neurol Brain Dis 3(1): 13- 17.

DOI: $10.15436 / 2377-1348.16 .019$

Keywords: Migraine; Intensity of pain; Frequency of crises; Ultra-micronized Palmitoylethanolamide (umPEA)

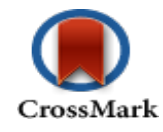

immune system, participate in numerous physiological and pathophysiological conditions.

The emergence of a local inflammatory response in the meninges is widely viewed as a potential contributor to the activation and sensitization of meningeal nociceptors during migraine ${ }^{[2]}$. Activation of resident immune cells such as mast cells, which are a prominent feature of the intracranial meninges, is likely to serve as a critical step in promoting enhanced excitability of meningeal nociceptors ${ }^{[3,4]}$.

Activation and sensitization of primary afferent meningeal nociceptive neurons, the peripheral arm of the trigem- 
inal-vascular system, appears to constitute one of the earliest events promoting the intracranial pain of migraine. These observations thus posit that meningeal mast cells, by virtue of their proximity both to meningeal blood vessels and nociceptive axons, may release a host of pro-inflammatory/algesic mediators responsible for the vasodilatatory phase of migraine associated with throbbing pain ${ }^{[3,5]}$. These data suggest that controlling meningeal nociceptor excitability might be achieved by acting on mast cell activation.

Palmitoylethanolamide (PEA), an endogenous fatty acid amide, is a congener of the endocannabinoid anandamide and belongs to a class of signaling lipids, the N-acylethanolamines. PEA is reported to exert anti-allodynic and anti-hyperalgesic effects by down-modulating both microglial and mast cell activity ${ }^{[6-8]}$. Through control of these targets, PEA might act as a disease-modifying rather than a symptom-modifying agent, since it exerts its effects on the "roots of the problem", i.e., on cellular targets involved in the generation and maintenance of pain $^{[8,9]}$. Based on this evidence, the present study was carried out to verify PEA efficacy in patients affected by migraine. For this we used an ultramicronized form of PEA (umPEA), which has been shown to display superior oral efficacy compared to non-micronized PEA ${ }^{[10]}$

\section{Patients and Methods}

Fifty patients with episodic migraine as defined by the International Headache Society were recruited from among those referring to the Headache Center Istituto Clinico Citta' di Brescia. All patients who were asked to participate in this study were informed in writing of its purpose; those agreeing to take part provided their written consent, in accordance with the Declaration of Helsinki; the scientific board of Istituto Clinico Citta' di Brescia was also informed. This observational study was carried out according to Good Clinical Practice.

Selection criteria for patients participating in this study: aged between 18 and 65 years; suffering from migraine without aura, according to the International Headache Society; suffering from migraine for at least 6 months with a monthly crisis frequency from 3 to 8 and with the presence of headache from 4 to12 days per month. Patients also were not to have taken other preventive therapies for migraine. Excluded from the study were patients: with psychiatric co-morbidity; suffering from other types of acute or chronic pain; with concomi-tant diagnosis of epilexia, kidney failure, neurological or oncological diseases or pregnancy.

The primary endpoint of the study was to evaluate, over the three-month observation period, reduction in headache days per month. The secondary endpoint was to assess duration, intensity, pain crisis and number of times analgesics were used per month. Additional secondary endpoints were the degree of patient satisfaction with analgesic therapy, response to trigger factors and improvement of thermographic patterns.

Eligible patients received umPEA, (Food for Special Medical Purposes Normast ${ }^{\circledR}$, Epitech Group, Saccolongo, Italy) microgranules, $600 \mathrm{mg}$ sublingual administration twice daily for 3 months.

At the enrollment visit diagnosis was confirmed also by thorough neurological examination. Before starting umPEA treatment (baseline) patients received instructions on how to re- cord in a headache diary on a day-to-day basis, migraine attacks. Patients had to register: i) migraine pain frequency, intensity and duration; ii) response to analgesics; iii) response to trigger factors. Each patient had also to indicate, by means of 0-5 scale, the self-perceived strength of trigger factors. At baseline (T0) and at treatment end (T1) all patients underwent instrumental evaluation by thermocamera (i3/i5/i7 FLIR) in order to assess changes in thermographic patterns. In particular, this was evaluated the mean temperature in the frontal area, which is thought to be a shunt between the ophthalmic artery, arising from the intracranial circle, and the superficial temporal artery, a branch of the external carotid.

This last parameter was selected based on the observation that, in the course of a migraine attack, hypothermia disappears after administration of sumatriptan ${ }^{[11]}$. Sumatriptan's beneficial effect on pain relief is associated with the disappearance of the cold patch when performing infrared (thermocamera) analysis. The mechanism underlying the disappearance of the cold patch in migraine is presumed to be vasodilation of the cutaneous microcirculation that is under the control of the autonomic system ${ }^{[12]}$. These data have been confirmed in an earlier study showing that thermography is useful to monitor the clinical disease course; further, thermographic improvement closely parallels the clinical course of migraine ${ }^{[12]}$.

Safety was evaluated by measuring discontinuation rate of treatment and the occurrence of adverse events, serious and otherwise.

\section{Results}

The 50 patients enrolled in this study comprised 12 males and 38 females, with a mean age of $37.6 \pm 16.2$ years (range: $18-75$ years) and average headache duration of 21.4 \pm 14.1 years (range: 2-64 years). Only one of the 50 patients failed to complete the study. This patient, a 60 -year-old female, withdrew after 1 month of treat-ment due to migraine worsening that disappeared after discontinuation of treatment. None of the patients com-pleting the study reported any treatment-related adverse events. At study start (baseline, T0) all the enrolled patients, except one, were undergoing standard analgesics, anti-inflammatory or triptan therapies for migraine and continued to take the same drugs together with umPEA. Due to the non-normal distribution of the data, statis-tical analysis was performed using the Wilcoxon test.

Days per month with migraine

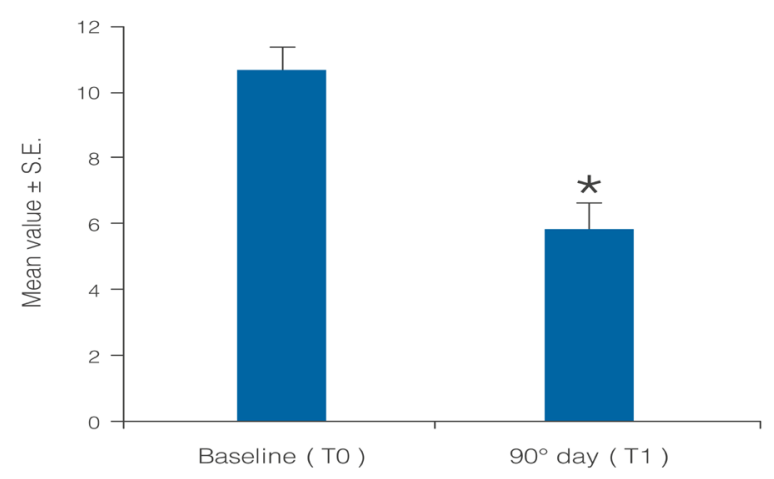

Figure 1: Decrease of days per month with migraine after 90 days of treatment with umPEA. ${ }^{*} \mathrm{p}<0.0001$ 
Statistical analysis showed that the observed parameters significantly improved at the end of the study period with umPEA treatment. The number of days per month with migraine decreased from a baseline average of $10.6 \pm 0.7$ to a value of 5.8 \pm 0.7 at treatment end (Figure 1; $<<0.0001$ ); this significant decrease of days with migraine was observed in $77.5 \%$ (38) of the patients, while the number of days was almost unchanged in the remaining $22.4 \%$ (11). Migraine intensity diminished in $71.4 \%$ of patients (35), and remained unchanged only in $28 \%$ (14). The baseline migraine severity that forced 28 out of 35 improved patients to stay in bed was considerably reduced, and did not minimally affect normal work activities. The difference in average value of pain intensity T1 vs T0 is shown in Figure. 2 and is statistically significant $(p<0.0001)$. Monthly frequency of crises diminished significantly $(\mathrm{p}<0.0001)$; this improvement was observed in $69,3 \%$ of patients (34) while for 15 patients $(30,6 \%)$ the number of crises per month remained unchanged. The mean value of crisis frequency changed from $6.5 \pm 0.8$ (T0) to $3.6 \pm 0.6$ (T1) (Figure. 3). Accordingly, the duration in days of migraine was notably $(\mathrm{p}<0.008)$ reduced (Figure. 4$)$. These relevant improvements occurred simultaneously with a significant decrease $(p<0.0001)$ in the number of analgesics taken over the 3-month treatment with umPEA (Figure. 5). Of the 49 patients who completed the study, $12(24 \%)$ continued to consume the same number of analgesics, $29(59,1 \%)$ had remarkably diminished the number of analgesics, while $8(16,3 \%)$ completely abandoned their intake of any drug for migraine. At the same time the patients showed a greater $(\mathrm{p}<0.0002)$ satisfaction with analgesics (Figure. 6). Furthermore, in 34 of the patients $(72,3 \%)$ the complexity of the accompanying symptoms was less severe and, at treatment end, a relevant reduction of the response to trigger factors was reported. The latter effect was probably due to cortical excitability and central sensitization reduction.



Figure 2: Mitigation of migraine pain intensity after 90 days of treatment with umPEA. ${ }^{*} \mathrm{p}<0.0001$

Thermo graphic examination at baseline showed the presence of right side hypothermia in 24 patients, and left side hypothermia in the other 25 patients. At the end of treatment all patients showed a reduction of hypothermia. In fact the Delta-Thermic (Delta-T), observed by infrared imaging in the middle area between the ophthalmic-frontal artery (belonging to the circle of the internal carotid artery) and temporal artery (branch of the external carotid artery) showed a decrease $>70 \%$. No serious adverse events were noted.

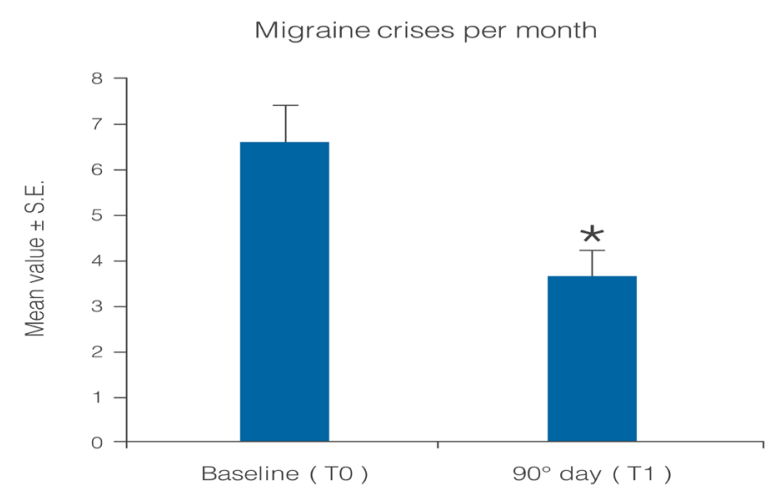

Figure 3: Reduction of migraine crisis number per month over a 90-day treatment period. ${ }^{*} \mathrm{p}<0.0001$.

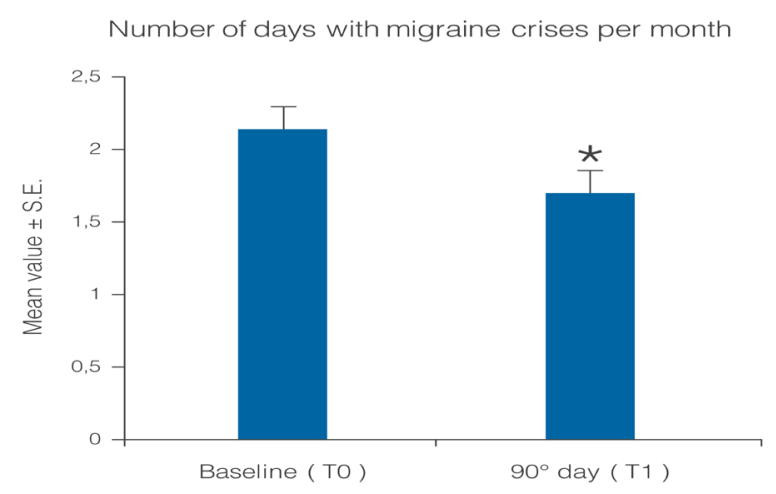

Figure 4: Number of days with migraine crises per month before and after treatment with umPEA. $* \mathrm{p}<0.008$.

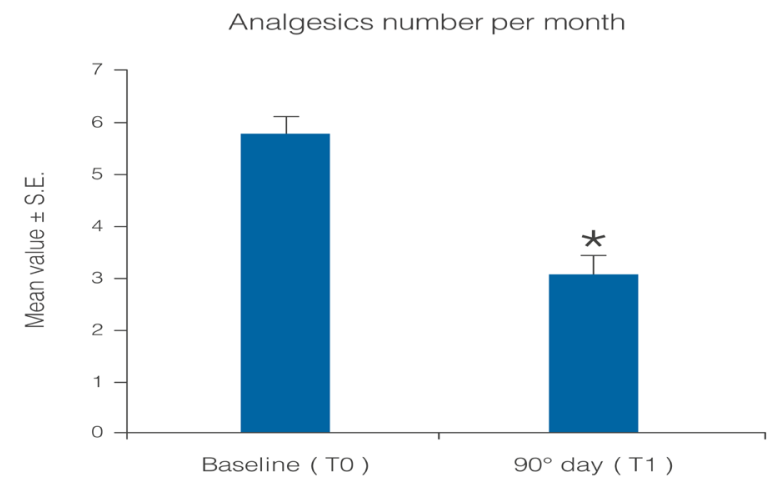

Figure 5: Reduction of number of analgesics taken per month over the treatment period. ${ }^{*} \mathrm{p}<0.0001$.

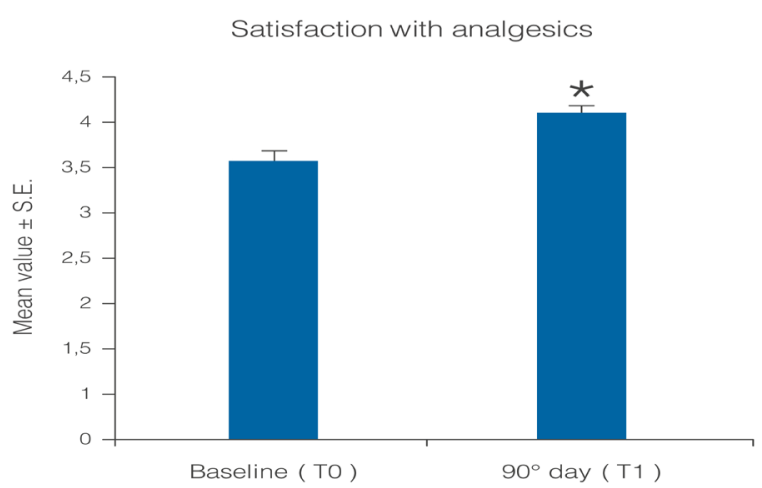

Figure 6: Patient satisfaction with analgesics before and after the treatment period. $* \mathrm{p}<0.0001$. 


\section{Discussion}

The results of this study highlight the safety and efficacy of treatment with umPEA in migraine. Symptoms were considerably improved in most of the patients treated, while the remaining patients were unchanged. In particular, two very interesting points merit highlighting, and are related to each other: the highly significant reductions in crisis frequency evaluated by day number per month (Figure. 1$)(p<0.0001)$ in the majority of patients (around $50 \%$ or more) and the Delta-T reduction observed by thermographic imaging ( $>70 \%$ of patients). These two parameters, which appeared after a short period of therapy, strengthen the idea that umPEA treatment induces not only a transitory and temporary desensitization of peripheral receptors, but also brings about a modification that persists over time - thus favoring long-term clinical benefit. As reported by patients, the reduced use of analgesics and especially the reduction of the response to trigger factors support the above evaluation.

These results are consistent with recent evidence showing that PEA levels are increased in the cerebral spinal fluid of patients with chronic migraine ${ }^{[13]}$; that could be interpreted as a physiological mechanism counteracting accelerated AEA catabolism in these pathological chronic pain conditions. These data may reflect impairment of the endocannabinoid system in these patients that can be prevented by administration of exogenous mo-lecules active on this system ${ }^{[13]}$.

These findings support the importance of a therapy intended to mitigate the inflammatory process due to production of mediators having a crucial role in the pathogenesis of migraine, as these agents contribute to the activation of meningeal nociceptors. It has been hypothesized that neurogenic inflammation in the meninges may be involved in pain generation or maintenance through a vicious cycle accompanying migraine $\operatorname{attacks}^{[14]}$. In numerous experimental models of inflammation and/or pain, PEA inhibits inflammatory processes ${ }^{[15]}$ through the regulation of mast cell degranulation ${ }^{[16,17]}$, thereby producing anti-nociceptive ${ }^{[18]}$ and neuroprotective ${ }^{[19]}$ effects. These actions are accompanied by changes in nitric oxide production ${ }^{[20]}$, neutrophil influx ${ }^{[21]}$, and expression of pro-inflammatory proteins such as inducible nitric oxide synthase and cyclooxygenase- $2^{[22]}$. PEA appears to exert its anti-inflammatory and anti-allodynic effects not only by modulating mast cell but also microglial activity $^{[23]}$. Recent evidence recognizes the involvement of microglia through the release of proinflammatory cytokines, in promoting the cortical spreading depression which, when recurrent, may play a role in the appearance of migraine ${ }^{[24]}$

In terms of PEA molecular target(s), this fatty acid amide is known to act as a ligand for the nuclear receptor peroxisome proliferator-activated receptor- $\alpha^{[25]}$. Furthermore, PEA potentiates anandamide actions in a cannabinoid $\mathrm{CB} 2$ receptor antagonist-sensitive fashion (while having itself no appreciable affinity for either CB1 or CB2 receptors) as well as anandamide desensitization of the transient receptor potential cation channel subfamily V member 1 (TRPV1) ("entourage" effect). Direct activation and desensitization of TRPV1 are also important mechanisms in the modulation of pain signaling ${ }^{[26,27]}$. Even the potentiating effects of PEA on anandamide vasorelaxant responses might be considered as part of an 'entourage' effect. The 'entourage' effect also provides support for an important function of TRPV1 in anandamide signaling in the circulation. PEA also elicits vasorelaxation per se and therefore might represent an important vasomodulator ${ }^{[28]}$. Conceivably, these effects could be invoked to explain our observations regarding the disappearance of hypothermia observed at the end of treatment with umPEA.

\section{Conclusion}

Despite the limitations of the study due to its open-label nature, our data allow the specialist to consider umPEA a valid molecule helpful as migraine preventive therapy especially in view of its excellent safety profile. For this purpose, it would be desirable to investigate with further clinical trials the umPEA effect in preventing migraine either given alone or in combination with other molecules.

Acknowledgments: No funding or sponsorship was received for this study. All authors discussed the results and commented on the manuscript. All named authors meet the International Committee of Medical Journal Editors criteria for authorship for this manuscript, take responsibility for the integrity of the work as a whole and have given final approval for the version to be published.

Conflict Of Interest: Giorgio Dalla Volta Paola Zavarize, Gaelle FK Ngonga and Daniela Carli declare no conflict of interest

Compliance with Ethics Guideline: All procedures followed were in accordance with the ethical standards of the responsible committee on human experimentation (institutional and national) and with the Helsinki Declaration of 1975, as revised in 2000 and 2008. Informed consent was obtained from all patients for being included in the study.

\section{References}

1. Moskowitz, M.A. Defining a pathway to discovery from bench to bedside: the trigeminovascular system and sensitization. (2008) Headache 48(5): 688-690.

2. Waeber, C., Moskowitz, M.A. Migraine as an inflammatory disorder. (2005) Neurology 64(10 Suppl 2): S9-15.

3. Levy, D., Burstein, R., Strassman, A.M. Mast cell involvement in the pathophysiology of migraine headache: A hypothesis. (2006) Headache 46(Suppl 1): S13-S18.

4. Levy, D. Migraine pain, meningeal inflammation, and mast cells. (2009) Curr Pain Headache Rep 13(3): 237-240.

5. Theoharides, T.C., Donelan, J., Kandere-Grzybowska, K., et al. The role of mast cells in migraine pathophysiology. (2005) Brain Res Brain Res Rev 49(1): 65-76.

6. Hansen, H.S. Palmitoylethanolamide and other anandamide congeners. Proposed role in the diseased brain. (2010) Exp Neurol 224(1): $48-55$.

7. Cerrato, S., Brazis, P., della Valle, M.F., et al. Effects of palmitoylethanolamide on immunologically induced histamine, PGD2 and TNFalpha release from canine skin mast cells. (2010) Vet Immunol Immunopathol 133(1): 9-15.

8. Skaper, S.D., Facci, L., Fusco, M., et al. Palmitoylethanolamide, a naturally occurring disease-modifying agent in neuropathic pain. (2014) Inflammopharmacology 22(2): 79-94.

9. Di Cesare Mannelli, L., D’Agostino, G., Pacini, A., et al. Palmitoylethanolamide is a disease-modifying agent in peripheral neuropathy: pain relief and neuroprotection share a PPAR-alpha-mediated mechanism. (2013) Mediators Inflamm 2013: 328797.

10. Impellizzeri, D., Bruschetta, G., Cordaro, M., et al. Micronized/ 
ultramicronized palmitoylethanolamide displays superior oral efficacy compared to nonmicronized palmitoylethanolamide in a rat model of inflammatory pain. (2014) J Neuroinflammation 11: 136.

11. Dalla Volta, G., Griffini, S., Pezzini, A. Influence of sumatriptan on the autonomic system during migraine attacks. (2006) J Headache Pain 7(2): 116-117.

12. Dalla Volta, G., Anzola, G.P., DiMonda, V. The disappearance of the "cold patch" in recovered migraine patients: thermographic findings. (1991) Headache 31(5): 305-309.

13. Sarchielli, P., Pini, L.A., Coppola, F., et al. Endocannabinoids in chronic migraine: CSF findings suggest a system failure. (2007) Neuropsychopharmacology 32(6): 1384-1390.

14. Williamson, D.J., Hargreaves, R.J. Neurogenic inflammation in the context of migraine. (2001) Microsc Res Tech 53(3): 167-178.

15. Mazzari, S., Canella, R., Petrelli, L., et al. N-(2-hydroxyethyl)hexadecanamide is orally active in reducing edema formation and inflammatory hyperalgesia by down-modulating mast cell activation. (1996) Eur J Pharmacol 300(3): 227-236.

16. Aloe, L., Leon, A., Levi-Montalcini, R. A proposed autacoid mechanism controlling mastocyte behaviour. (1993) Agents Actions 39: C145-C147.

17. Bettoni, I., Comelli, F., Colombo, A., et al. Non-neuronal cell modulation relieves neuropathic pain: efficacy of the endogenous lipid palmitoylethanolamide. (2013) CNS Neurol Disord Drug Targets 12(1): 34-44.

18. Calignano, A., La Rana, G., Piomelli, D. Antinociceptive activity of the endogenous fatty acid amide, palmitylethanolamide. (2001) Eur J Pharmacol 419(2-3): 191-198.

19. Ahmad, A., Genovese, T., Impellizzeri, D., et al. Reduction of ischemic brain injury by administration of palmitoylethanolamide after transient middle cerebral artery occlusion in rats. (2012) Brain Res 1477: 45-58.
20. Ross, R.A., Brockie, H.C., Pertwee, R.G. Inhibition of nitric oxide production in RAW264.7 macrophages by cannabinoids and palmitoylethanolamide. (2000) Eur J Pharmacol 401(2): 121-130.

21. Farquhar-Smith, W.P., Rice, A.S. A novel neuroimmune mechanism in cannabinoid-mediated attenuation of nerve growth factor-induced hyperalgesia. (2003) Anesthesiology 99(6): 1391-1401.

22. Costa, B., Comelli, F., Bettoni, I., et al. The endogenous fatty acid amide, palmitoylethanolamide, has anti-allodynic and anti-hyperalgesic effects in a murine model of neuropathic pain: involvement of $\mathrm{CB}(1)$, TRPV1 and PPARgamma receptors and neurotrophic factors. (2008) Pain 139(3): 541-550.

23. Luongo, L., Palazzo, E., de Novellis, V., et al. Role of Endocannabinoids in Neuron-Glial Crosstalk. (2010) Open Pain J 3: 29-36.

24. Pusic, K.M., Pusic, A.D., Kemme, J., et al. Spreading depression requires microglia and is decreased by their M2a polarization from environmental enrichment. (2014) Glia 62(7): 1176-1194.

25. Lo Verme, J., Fu, J., Astarita, G., et al. The nuclear receptor peroxisome proliferator-activated receptor-alpha mediates the anti-inflammatory actions of palmitoylethanolamide. (2005) Mol Pharmacol 67(1): $15-19$.

26. Ambrosino, P., Soldovieri, M.V., Russo, C., et al. Activation and desensitization of TRPV 1 channels in sensory neurons by the PPAR $\alpha$ agonist palmitoylethanolamide. (2013) Br J Pharmacol 168(6): 1430-1444. 27. De Petrocellis, L., Harrison, S., Bisogno, T., et al. The vanilloid receptor (VR1)-mediated effects of anandamide are potently enhanced by the cAMP-dependent protein kinase. (2001) J Neurochem 77(6): $1660-1663$.

28. Ho, W.S., Barrett, D.A., Randall, M.D. "Entourage" effects of $\mathrm{N}$-palmitoylethanolamide and $\mathrm{N}$-oleoylethanolamide on vasorelaxation to anandamide occur through TRPV1 receptors. (2008) Br J Pharmacol 155(6): 837-846.
Online ISSN: $2377-1348$

Journal Title: International Journal Neurology and Brain Disorders Journal Short Name: Int J Neurol Brain Disord
Ommega Online Publishers

E-mail: neurology@ommegaonline.com

Website: www.ommegaonline.org 